\title{
Analyzing Missense Mutations of the MAPT/Tau Gene to Predict Variant Pathogenicity in Alzheimer's Disease
}

\author{
Tejas Shah \\ Hopkinton High School, 90 Hayden Rowe, Hopkinton, MA, 01748, USA; tshah@hillers.org
}

\begin{abstract}
Alzheimer's Disease (AD) continues to affect millions of people and is a leading cause of death in the United States. This stands true mainly in light of the fact that the underlying mechanisms of AD are unclear and there is no effective method of preventing neurodegeneration. The Tau protein, however, has shown to play an important role in the pathogenesis of the disease. Mutations in the MAPT/Tau gene can lead to complications in the functionality of Tau, potentially fast-tracking AD development. The goal of this study was to investigate potential missense mutations in this gene in order to identify those that were most pathogenic. Since missense mutations carry unknown effects on protein function, they were closely examined. Several amino acid (AA) changes such as hydrophobicity, charge, and polarity were investigated. Of 109 reported missense mutations, 72 resulted in significant AA changes. Due to their unknown effects, additional criteria such as AA conservation and mutation location with respect to tubulin-binding domains were also factored in to investigate overall impact. Through this comprehensive methodology, three mutations that were more likely to carry deleterious effects and potentially causing AD susceptibility in individuals with these alterations were identified.

KEYWORDS: Biomedical and Health Sciences; Genetics and Molecular Biology of Disease; Alzheimer's Disease; Tau; Bioinformatics.
\end{abstract}

\section{Introduction}

Alzheimer's Disease (AD) is a progressive neurodegenerative disorder associated with memory loss and dementia. ${ }^{1}$ At least 5.8 million individuals over the age of 65 within the United States live with the illness today and there were more than 120,000 recorded mortalities in 2018 alone, making it the sixth leading cause of death in the nation. ${ }^{2}$ There is no known cure for the disorder as of now, but the frequency of cases is rising exponentially per decade, making it increasingly important to further understand the underlying causes of the illness.

During initial stages of progression, $\mathrm{AD}$ targets areas of the brain that are responsible for controlling thought, memory, and language, including the hippocampus and the entorhinal cortex, making these structures vulnerable to atrophy. ${ }^{3}$ This results in an inability to recall basic information such as recent events or familiar names. As the disease progresses, the patient may be unable to recognize friends and family. They may also have trouble with verbal communication including reading, writing, and in some cases, speaking. During late-stage $\mathrm{AD}$, the patient will need around the clock care, and this can be very demanding of family members and/or caregivers. ${ }^{4}$ The patient will eventually lose the ability to carry out essential tasks such as bathing, eating, or dressing and will be in constant need of attention. ${ }^{5}$

The exact cause of $\mathrm{AD}$ is unknown, but genetics is speculated to be one of the most prominent factors to be associated with the illness. ${ }^{6}$ Mutations in the Microtubule Associated Protein Tau (MAPT/Tau) gene can affect the functioning of the Tau protein. In healthy brains, the protein is primarily responsible for microtubule assembly and construction. ${ }^{7}$ Recent studies have also shown that Tau plays a vital role in cellular signaling, synaptic plasticity, and genomic stability. However, under certain conditions, it can become insoluble, resulting in synaptic dysfunction and eventually, neural cell death, often referred to as tauopathies. This process occurs in a wide range of neurodegenerative disorders, including $\mathrm{AD}$ and Parkinson's. ${ }^{8}$ The amount of phosphate within the brain also has an impact on the behavior of the Tau protein. In healthy adults, the brain consists of 2 to 3 moles phosphate per mole of Tau, and its biological activity is suppressed by hyperphosphorylation. However, in the brains of Alzheimer's patients, Tau is hyperphosphorylated to approximately 3 to 4 times it should typically be. Because the function of the protein becomes compromised, abnormal Tau folding may occur, which can lead to a genesis of paired helical and straight filaments within neurons. ${ }^{9}$ This prompts the formation of neurofibrillary tangles and Tau accumulation within synapses and can lead to synaptic blockage, inhibiting cellular communication and resulting in cell death.

Genetic mutations always occur in cells but are often harmless. Occasional mutations in gene sequences that encode for crucial amino acids can, however, have severe repercussions as they may alter the way the protein functions altogether. This study looked at the various missense mutations of the MAPT/ Tau isoform 6 gene through the use of Geno2MP, an online software that searches a database of rare variants from exome sequencing data. Missense mutations were specifically targeted in this study due to their unknown effect on protein function and pathogenicity. Changes in hydrophobicity, charge, and polarity were determined, then analyzed through the addition of special criteria. The Clustal Omega server and NCBI 
were used to gauge AA conservation within the MAPT/Tau FASTA sequence in humans and other organisms including Caenorhabditis elegans (Ce), Danio rerio (Dr), Drosophila melanogaster (Dm), Mus musculus (Mm), Rattus norvegicus (Rn), and Xenopus tropicalis $(\mathrm{Xt})$. This would allow for the determination of the importance of each mutation and formulate a more accurate conclusion on mutation significance. In an effort to further improve the accuracy and validity of the analysis, the four tubulin-binding domains to which some of these altered amino acids belong were also identified through use of databases such as MARRVEL (Model organism Aggregated Resources for Rare Variant ExpLoration) and NCBI. Typically, AAs within these domains are known to be essential for protein functionality therefore mutations taking place here were more closely examined. A combination of all these factors were used while evaluating and identifying the most harmful missense mutations in the MAPT/Tau gene to see if they could potentially contribute to dementia and AD.

\section{Methods}

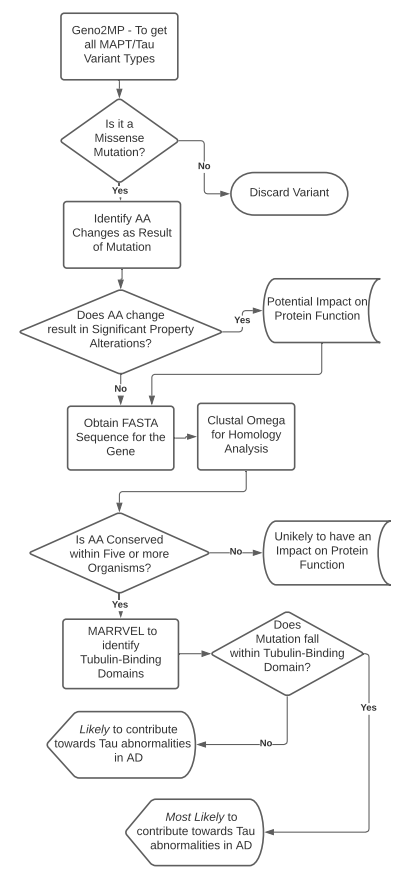

Figure 1: Workflow diagrams for identifying most deleterious mutations of the MAPT/Tau gene. Analysis was mutually exclusive.

\section{Identifying Missense Mutations in the MAPT/Tau gene:}

The online software, Geno2MP, was used to generate a list of all possible mutations and their properties within the MAPT/Tau isoform 6 gene (NP_001116538.2). Geno2MP is an online tool that searches a database for rare variants from exome sequencing data linked to phenotypic information from a variety of Mendelian gene discovery projects. The database contains information from more than 19,000 individuals that includes both persons affected by Mendelian conditions and unaffected relatives of these persons. A table containing all possible MAPT/Tau isoform 6 mutations was generated and then exported to Microsoft Excel, where further analysis took place. Data was then filtered by looking at the "fxnAnnotation" column, where only missense mutations were included, while the rest were discarded. Missense mutations were closely examined because their effects on the protein are still unclear as they could carry negative or negligible effects. Identifying these mutations would later allow for a closer look at what effects they can inflict on the overall functioning of the Tau protein. They were evaluated in terms of resulting AA property changes, AA conservation, and their locations with respect to tubulin-binding domains. Mutations that satisfied these criteria (Figure 2) were deemed likely to alter the function of the Tau protein and contribute towards AD pathogenesis.

\section{Identifying specific Amino Acid (AA) changes:}

Upon studying the "hgvsProteinVar" column in the table, specific AA changes, and their locations in the gene were identified. Following this process, the effects of these changes were then noted. The three properties that were investigated in this analysis were hydrophobicity, polarity, and charge. These properties were confirmed true with verified online tables and sources. A change in any of these factors as a result of a missense mutation could have potentially impaired the function of the Tau protein. If a change in AA property took place, the mutation was noted as significant in the next column. All 109 mutations were still taken to the next step of analysis even though some were found insignificant.

\section{Gauging Gene Conservation :}

The FASTA sequence of the MAPT/Tau isoform 6 gene in Homo Sapiens was obtained through NCBI and recorded. In order to determine conservation amongst Homo sapiens (Hs) and the other organisms, the FASTA sequences of Caenorhabditis elegans (Ce), Drosophila melanogaster (Dm), Danio rerio (Dr), Mus musculus (Mm), Rattus norvegicus (Rn), and Xenopus tropicalis (Xt) were also taken note of. These sequences (including $\mathrm{Hs}$ ) were entered into Clustal Omega, an online tool that aligned the AA sequences to test the level of AA conservation amongst different organisms. With this alignment data, each $\mathrm{AA}$ from the table was investigated to see which were shared with the other organisms at their respective locations. If organisms shared one or more of the same $\mathrm{AA}$ in the same position, it was marked as conserved at that location.

\section{Finding Altered AAs in Tubulin-Binding Domains :}

The MAPT/Tau protein has four tubulin-binding domains. For each domain, AA sequences were found through the MARRVEL/DIOPT software for MAPT/Tau isoform $\mathrm{X} 1$. Typically, AAs within these domains are known to be essential for protein functionality so mutations taking place here were closely examined. These AA sequences were then identified within the MAPT/Tau isoform 6 gene and their beginnings and ends were defined. Then, it was investigated whether any of the reported mutations took place within the tubulin-binding domains.

\section{- Results}

\section{Reported MAPT/Tau mutations :}

The MAPT/Tau gene on chromosome 17 has a reported 109 missense mutations that were found through the $\mathrm{Ge}-$ no2MP online database. Each of these reported muta 
tions were from mRNA NM_001123066.3, the MAPT/ Tau isoform 6 gene was used as a reference in this study. See Supplemental Table 1, for full list of identified missense mutations. These mutations were analyzed throughout the duration of this study.

\section{Analysis of $A$ A changes :}

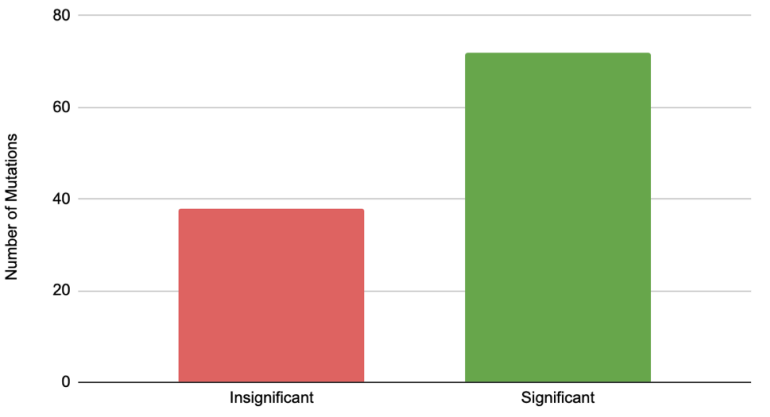

Figure 2: · Significance of Mutations - Of the 109 missense mutations reported, 72 were found to be significant based solely on resulting property alterations in hydrophobicity, polarity, and charge. 37 were said to be insignificant as they resulted in no changes in the traits listed above. For full list of mutation property changes, see Supplemental Table 2.

Missense mutations occur at a nucleotide level and can therefore lead to potential AA changes, causing certain alterations in protein function. The first mutation reported was $\mathrm{G}>\mathrm{A}$ (guanine to adenine), which led to a change of the AA arginine (Arg) to histidine (His), (Supplemental Table 2). Three specific properties were investigated while evaluating AA changes that included hydrophobicity, polarity, and charge. Each AA property was determined and confirmed true through the use of verified sources. ${ }^{10}$ Mutation \#1 did not cause any changes in these properties, so it was marked as insignificant. The second mutation reported was $\mathrm{G}>\mathrm{T}$ (guanine to thymine) and caused an AA change of arginine (Arg) to leucine (Leu). However, unlike the previous mutation, Mutation \#2 was found to bring changes to the AA (Suplementel Table 2, Column 5), so it was marked as significant. This process was repeated several times in order to determine each mutation's theoretical impact on the protein and all findings are listed (Supplemental Table 2). Of the 109 identified missense mutations, 72 were found to alter the respective AA in regard to hydrophobicity, polarity, or charge and were therefore deemed significant (Figure 2).

AA Conservation amongst other organisms :

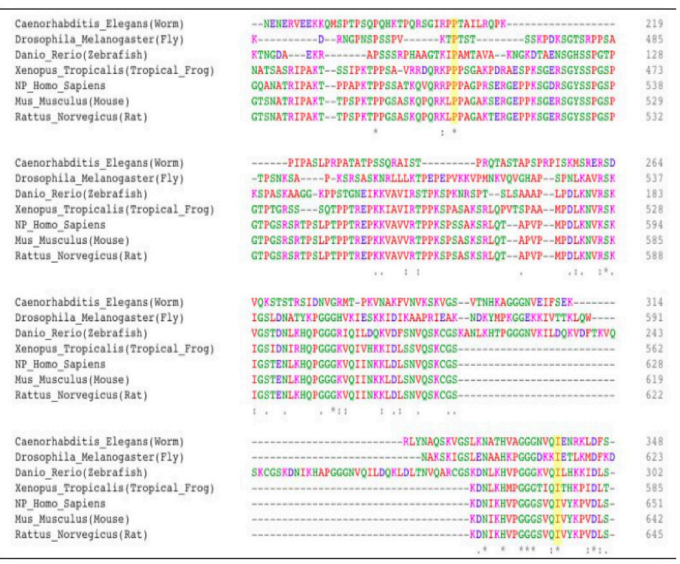

Figure 3: Segment of Aligned FASTA sequence of Ce, Dm, Dr, Hs, Mm, $\mathrm{Rn}$, and $\mathrm{Xt}$ - Image captured from Clustal Omega. Red letters signify small and hydrophobic AAs, blue represents acidic AAs, magenta letters represent basic AAs, and green represents AAs with a hydroxyl, sulfhydryl, or amine functional group. An asterisk (*) indicates positions which have a single, fully conserved residue (See highlighted columns for fully conserved AAs from mutation list), a colon (:) indicates conservation between groups of strongly similar properties, a period (.) indicates conservation between groups of weakly similar properties.

Conservation is an important feature of AAs in a protein as it can be very useful for evaluating the cruciality of certain missense mutations. An essential AA is expected to be highly conserved amongst organisms, while relatively less important AAs are not as conserved. Significant changes in conserved AAs are more likely to alter the functioning of the protein because crucial AAs are at greater risk of resulting in property changes. In order to analyze the most important AA changes, then aligned the MAPT/Tau FASTA sequence of Caenorhabditis elegans (Ce), Drosophila melanogaster (Dm), Daniorerio (Dr), Mus musculus (Mm), Rattus norvegicus (Rn), Xenopus tropicalis $(\mathrm{Xt})$, and Homo sapien (Hs) using the online software Clustal Omega (Figure 3). Of the 109 mutations, 83 were found to be conserved in at least one or more of the listed organisms (Supplemental Table 3), however only Mutation \#91, Mutation \#92, and Mutation \#102 were conserved in all five (Table 1)

Table 1: Fully Conserved AAs from Mutation List. Within the 109 missense mutations, it was found that only three AAs were fully conserved amongst all tested organisms. For a full list of AA conservation, see Supplemental Data Table 3, Appendix 1.

\begin{tabular}{|c|c|c|c|c|}
\hline Mutation \# & hgvsProteinVar & hgvsAlleleChange & AA Conserved? & AA Conserved in... \\
\hline 91 & p.(P512S) & C>T & Yes & Ce, Dm, Dr, Mm, Rn, Xt \\
\hline 92 & p.(P512H) & C>A & Yes & Ce, Dm, Dr, Mm, Rn, Xt \\
\hline 102 & p.(1643T) & T>C & Yes & Ce, Dm, Dr, Mm, Rn, Xt \\
\hline
\end{tabular}

\section{Mutations Within Tubulin-Binding Domains :}

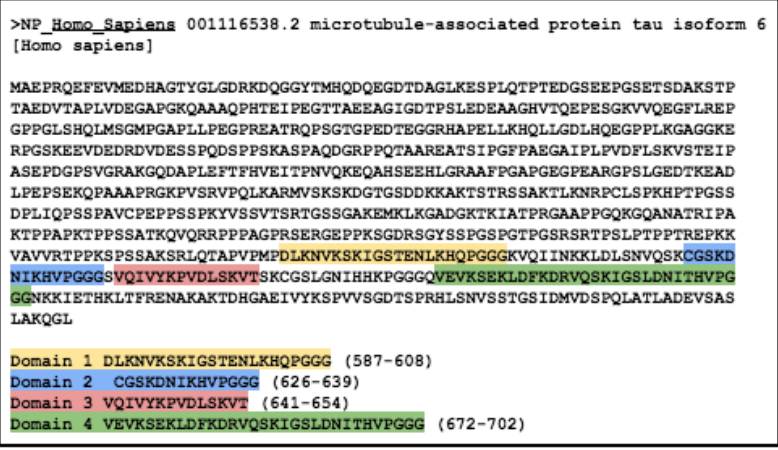

Figure 4: The four tubulin-binding domains of MAPT/Tau isoform 6 marked in its FASTA sequence (Source: NCBI/Protein). Domains are represented with corresponding colors. Domain 1 (587-608) is highlighted in yellow; Domain 2 (626-639) is highlighted in blue; Domain 3 (641-654) is highlighted in red; and Domain 4 (672-702) is highlighted in green.

The MAPT/Tau protein has four tubulin-binding domains, highlighted in Figure 3. It was found that only $\mathrm{Mu}-$ tation \#101, \#102, and \#103 fall into one of these domains (Table 2). Mutations within these domains were explicitly inspected because AAs found within functional domains are known to be more essential for protein functionality. 
Table 2: Mutations that took place in tubulin-binding Domains. Of the 109 different missense mutations, it was determined that only three (101 103) took place in the tubulin-binding domain. Mutation \#101 occurred in Domain 2, Mutation \#102 occurred in Domain 3, and Mutation \#103 occurred in Domain 4. None of the recorded missense mutations took place in Domain 1.

\begin{tabular}{|c|c|c|c|}
\hline Mutation\# & hgvsAlleleChange & hgvsProteinVar & Domain of the Mutation \\
\hline 101 & G>A & p.(V635I) & Domain 2 \\
\hline 102 & T>C & p.(I643T) & Domain 3 \\
\hline 103 & G>A & p.(V698I) & Domain 4 \\
\hline
\end{tabular}

\section{- Discussion}

In this study, an approach to predict the pathogenicity of possible MAPT/Tau missense mutations was developed. It was found that depending on the type of mutation and its location in the gene, the overall effects may vary. AA changes were analyzed in the context of polarity, hydrophobicity, charge, and conservation amongst various organisms. Specific AA changes were investigated based on their position in the different functional domains of the protein. This information could be used to predict the probability of having a damaged or impaired Tau protein and could further help in predicting the risk of $\mathrm{AD}$ occurrence in individuals. This general bioinformatics approach can be replicated to predict the occurrence of other genetic diseases/illnesses by utilizing the gene sequence of an affected protein. This technique could be used to predict certain cancers with a known genetic component and other proteins that play a role in $\mathrm{AD}$ such as amyloid beta.

A total of 109 missense mutations were analyzed in the MAPT/Tau gene and 72 of them were determined to be significant based on changes in their corresponding AA (Figure. 1). Mutation \#101 (p.(V635I)), Mutation \#102 (p.(I643T)), and Mutation \#103 (p.(V698I)) were the only alterations that were found to occur within a tubulin-binding domain. Furthermore, Mutation \#102 was the only variant change determined to be both significant and fully conserved amongst these three. Significance in this context refers to property changes in AAs as a direct result of a mutation. To test for overall significance on protein function and the vitality of the AA, conservation was also evaluated. Since the AA Isoleucine was fully conserved at its location (p.643), Mutation \#102 is more likely to carry a deleterious effect on the overall functionality of the Tau protein. It is important here because based of this evidence, it can be concluded that Mutation \#102 will most likely alter AA function, potentially impairing the functionality of the protein.

Mutation \#91 (p.(P512S)) and Mutation \#92 (p.(P512H)) were also found to be both fully conserved and significant based on property changes. However, unlike Mutation \#102, neither belonged to a tubulin-binding domain. Because fully conserved AAs are more likely to alter the overall functionality of the protein and these mutations were determined to be significant, changes are more likely to carry through. Therefore, it was concluded that Mutation \#91 and \#92 also carry a high probability of damaging the Tau protein and can potentially lead to $\mathrm{AD}$.
Several other mutations were noted as significant as well, however they did not all meet the conservation threshold, nor did they belong to a tubulin-binding domain. Significant property changes alone, are not always enough to considerably alter protein functionality. This needs to be taken into account along with AA conservation to obtain a more accurate result as to which mutations can be most deleterious.

\section{Conclusion}

Three missense mutations that were likely to damage the functionality of the Tau protein were identified. Mutation \#91, \#92, and \#102 were fully conserved and brought changes to AA properties. Alterations as such in the MAPT/Tau gene could potentially increase the risk of developing Tau abnormalities and ultimately, AD. These specific changes can be detected early on through methods of gene analysis to predict $\mathrm{AD}$ occurrence and prevent or delay symptoms. By using this approach, there is a hope to pave the way for better understanding the underlying mechanisms of $\mathrm{AD}$ and predict its development in patients by looking at changes in relevant gene sequences.

\section{Supplemental Data :}

Supplemental Table 1: Geno2Mp Generated Missense Mutation list. It was discovered that there were 109 reported missense mutations in this specific gene- This table was found in the online database $\mathrm{Geno}_{2} \mathrm{MP}$. It includes Mutation Number (\#), Chromosome number (\#), Chromosome position (chrPos), Reference SNP cluster ID (rsID), Allele Change (hgvsAlleleChange), Type of Mutation (fxnAnnotation), mrnaAccession, and the AA change and as a result of an alteration at the numbered locations(hgvsProteinVar). The symbols included here are change (>), adenine (A), cytosine $(\mathrm{C})$, guanine $(\mathrm{G})$, thymine $(\mathrm{T})$, and position (p.). The mutation numbers shown here are constant throughout the entire analysis.

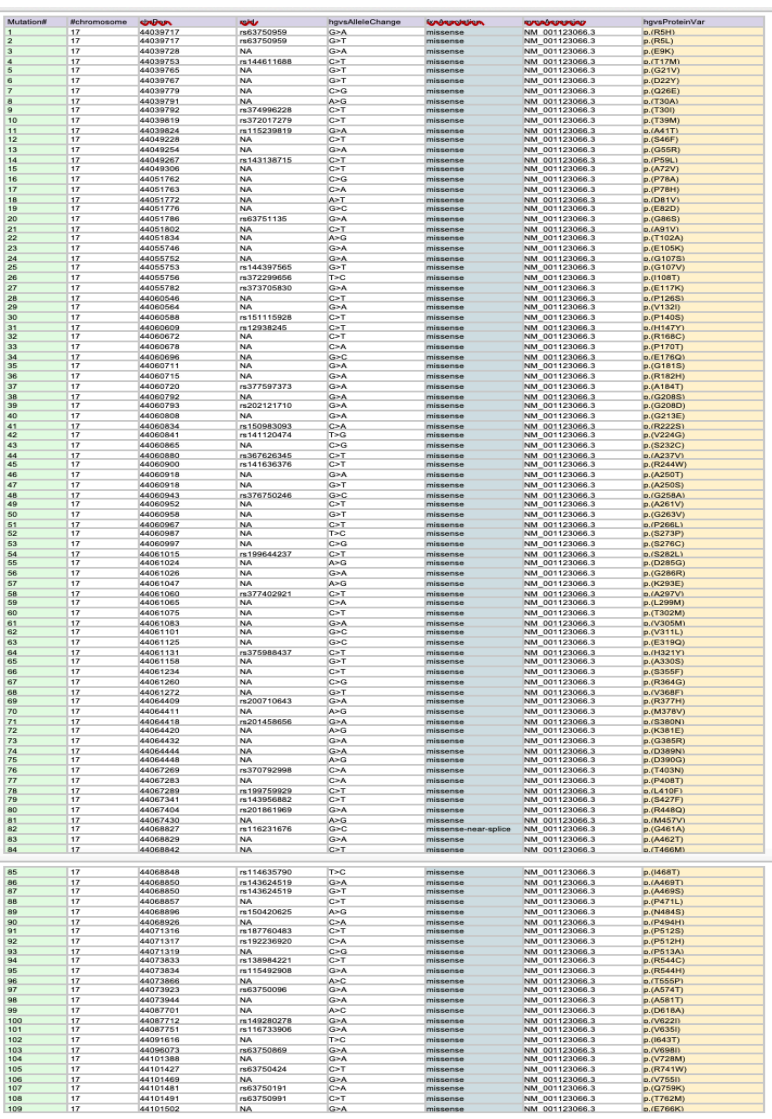


Supplemental Table 2. Mutation Property Changes. : The AA changes were evaluated based on polarity, hydrophobicity, and charge Specific AA changes are highlighted in yellow and corresponding property shifts are highlighted in blue. Mutation significance is specified by a green highlight, while insignificant mutations are marked in red. Columns without a highlight were generated by $\mathrm{Geno}_{2} \mathrm{MP}$, and the others were a result of the analysis.

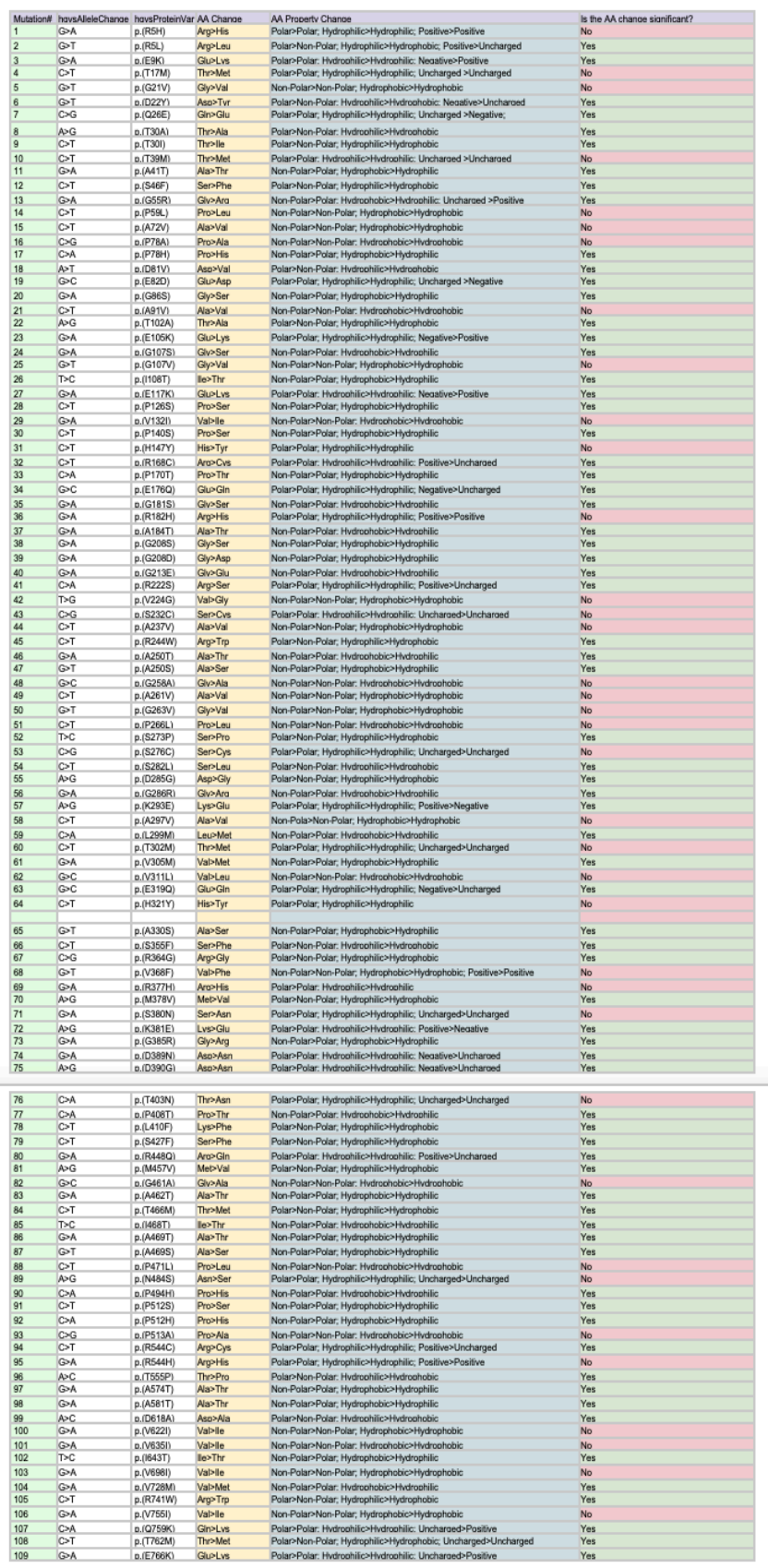

Supplemental Table 3. AA Conservation Table : Within the 109 mutated AAs, 83 were found to be conserved within at least one or more of the tested organisms. The table was filtered to only display rows in which the AAs were conserved. Organisms that share an AA at their corresponding locations are listed in the column, highlighted in blue. Rows in which AAs were fully conserved amongst all organisms are highlighted in bright yellow. Columns without a highlight were generated by $\mathrm{Geno}_{2} \mathrm{MP}$, while the others were created through the analysis.

\begin{tabular}{|c|c|c|c|c|c|c|c|c|}
\hline Mutationt & hgveprototion & Ir hgvaAdlele Chango & A Consoonod? & AA consorved in. & 5E p.(A297) & $C T$ & $Y_{e s}$ & $M m, R_{n}$ \\
\hline & 19.(IFH) & $G A$ & Yes & $M m, R n$ & 6сp. (T302M) & $C D$ & Yes & \\
\hline & $\begin{array}{l}2 p .(1.1521 \\
\text { s. (G21V) }\end{array}$ & $\begin{array}{l}G T \\
G T \\
G T\end{array}$ & $\begin{array}{l}\text { Yes } \\
\text { Yes }\end{array}$ & $\begin{array}{l}M_{m}, R_{n} \\
D m\end{array}$ & 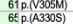 & $\begin{array}{ll}Q A A \\
Q T T\end{array}$ & $\begin{array}{l}\text { Yes } \\
\text { Yes }\end{array}$ & 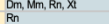 \\
\hline & 8p.(T30A) & $A>C$ & Yes & $M m, R n$ & 6є $\mathrm{p}(\mathrm{N}(36 \mathrm{68F})$ & & Yes & \\
\hline & 9p.(T301) & CT & Yes & $M m, R n$ & $6 \in \mathrm{p} \cdot(\mathrm{R} 37 \mathrm{H} H)$ & $G \curvearrowright A$ & Yes & $\mathrm{Mn}, \mathrm{Rn}, \mathrm{Dm}$ \\
\hline & 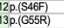 & ${ }_{G A}^{C D}$ & $\begin{array}{l}\text { Yes } \\
\text { Yes }\end{array}$ & 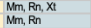 & 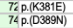 & $\begin{array}{l}A \geq G \\
G>A\end{array}$ & $\begin{array}{l}\text { Yes } \\
\text { Yys }\end{array}$ & $\begin{array}{l}x_{1} \\
M, R n\end{array}$ \\
\hline & 14p.(P59L) & OT & Yes & $\mathrm{Mm}, \mathrm{Rn}$ & $7 \in$ p. (T403N) & $C>A$ & Yes & \\
\hline & 15p.(A722) & & & & 77 . (P408T) & $C>A$ & Yes & $\mathrm{Ce}, \mathrm{Dm}, \mathrm{Mm}, \mathrm{Rn}$ \\
\hline & 16p.(P78A) & $\infty$ & Yes & $\mathrm{Dm}, \mathrm{Mn}_{\mathrm{n}} \mathrm{Rn}$ & $7 \in p,(L 410 F)$ & $C D$ & Yes & \\
\hline & 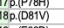 & $\begin{array}{l}C A \\
A>T\end{array}$ & $\begin{array}{l}\text { Yres } \\
\text { Yes }\end{array}$ & $\begin{array}{l}\mathrm{Dmm}, 1, \mathrm{~m}, \mathrm{Rn} \\
\mathrm{Mm}\end{array}$ & 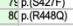 & 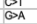 & $\begin{array}{l}\text { YYs } \\
\text { Yes }\end{array}$ & $\begin{array}{l}\text { Dmori, Mm, Rn } \\
\text { Mn, Rn }\end{array}$ \\
\hline & (19p, $(E 820)$ & $a x$ & Yes & $M m, R n$ & 81P.(M45TV) & $A>B$ & Yes & $\mathrm{D} ; \mathrm{Mm}, \mathrm{Rn}$ \\
\hline & 20p.(C865) & $G A$ & Yes & & $82 p$ (G461A) & $G \times C$ & Yes & $M n, B n$ \\
\hline & 21p.A991V & $C D$ & Yes & & 8: $p$. (A462T) & $Q A$ & Yes & $M m, R n, X t$ \\
\hline & 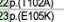 & $\frac{A \rightarrow G}{G A}$ & $\begin{array}{l}\text { Yes } \\
\text { Yos }\end{array}$ & $\begin{array}{l}D_{m}, M_{n}, R_{n}, X_{t} \\
M_{n}, R_{n}, x\end{array}$ & 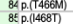 & CT & $\begin{array}{l}\text { Yes } \\
\text { Yess }\end{array}$ & $\begin{array}{l}M M_{1}, R n \\
M m, n n\end{array}$ \\
\hline & $24,(G 1075)$ & $Q A$ & Yes & $\mathrm{Mm}, \mathrm{Rn}, \mathrm{xt}$ & 8Ep.(A469T) & 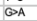 & Yes & $\mathrm{D} ; \mathrm{Mm}, \mathrm{Rn}$ \\
\hline & 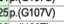 & $G T$ & Yes & $M_{m}, R_{n}, x_{t}$ & 8ip.(A46SS) & $G T^{2}$ & Yes & $\mathrm{D} ; \mathrm{Mm}, \mathrm{Rn}$ \\
\hline & 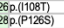 & $\begin{array}{l}T \times C \\
C P T\end{array}$ & $\begin{array}{l}\text { Yes } \\
\text { Yes }\end{array}$ & $\begin{array}{l}M M_{n}, R n \\
M m, R n\end{array}$ & 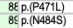 & $\underset{A>C}{D>C}$ & $\begin{array}{l}\text { Yes } \\
\text { Yes }\end{array}$ & $\begin{array}{l}\mathrm{M}, \mathrm{R} n \\
\mathrm{M}, \mathrm{R}, \mathrm{n}\end{array}$ \\
\hline & 299.N(1321) & $G A$ & Yes & $\mathrm{Dm}$ & 96 p.(P4944) & $C>A$ & Yes & $\mathrm{Ce}, \mathrm{Mm}, \mathrm{Rn}$ \\
\hline & 30p.p(P140S) & & Yes & $M m, R n$ & 91p.(P512S) & CT & Yes & $\mathrm{Ce}, \mathrm{Dm}, \mathrm{Dr}, \mathrm{Mm}, \mathrm{Rn}, \mathrm{X}$ \\
\hline & 31p.(141477) & CT & Yes & $R_{n}$ & gep.(P512H) & $C A A$ & Yes & $\mathrm{Ce}, \mathrm{Dm}, \mathrm{Dr}, \mathrm{Mm}, \mathrm{Rn}, \mathrm{X}$ \\
\hline & 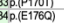 & $\begin{array}{l}C A \\
G C\end{array}$ & $\begin{array}{l}\text { Yes } \\
\text { Yes }\end{array}$ & $\begin{array}{l}M M_{n}, R_{n} \\
M m, R n\end{array}$ & 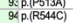 & $\begin{array}{l}D, G \\
C D T\end{array}$ & $\begin{array}{l}\text { Yess } \\
\text { Yes }\end{array}$ & 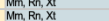 \\
\hline & (37.(A184T) & $G A$ & Yos & $M m, R_{n}$ & of $p$. (R544th) & $\theta A$ & Yes & $M M_{n}, B_{n}, x_{t}$ \\
\hline & 38p.(C2028S) & & Yes & $\mathrm{Mm}, \mathrm{R} \mathrm{n}$ & 9 ef $\mathrm{p}$.(T555P) & $A>C$ & Yes & $\mathrm{Ce}_{1} \mathrm{Mm}, \mathrm{Rn}, \mathrm{Xt}$ \\
\hline & 39p.(62030) & $G A$ & Yes & $M m, R n$ & gip.(A574T) & $G A$ & Yes & \\
\hline & 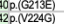 & ${ }_{T \supset G}^{G A}$ & $\begin{array}{l}\text { Yes } \\
\text { Yes }\end{array}$ & $\begin{array}{l}M M_{m}, R n, x t \\
M m\end{array}$ & 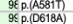 & $\underset{A \sim C}{Q A C}$ & $\begin{array}{l}\text { Yes } \\
\text { Yes }\end{array}$ & $\mathrm{Ce}, \mathrm{Dr}, \mathrm{Mr}, \mathrm{Rn}$, \\
\hline & & & Yes & $M n, R n$ & & $\theta A$ & Yes & $\mathrm{Ce}, \mathrm{Dr}, \mathrm{Mn}, \mathrm{Rn}, \mathrm{Xt}$ \\
\hline & 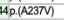 & OT & Yes & $M m, R n$ & 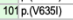 & $G A$ & Yes & $\mathrm{Ce}, \mathrm{Dr}, \mathrm{Mn}, \mathrm{Rn}$ \\
\hline & 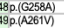 & $\begin{array}{l}G \times C \\
C D T\end{array}$ & $\begin{array}{l}\text { Yes } \\
\text { Yys }\end{array}$ & ${ }_{\mathrm{Rn}}^{\mathrm{Mn}, \mathrm{Rn}}$ & 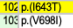 & $\begin{array}{l}T C C \\
G A A\end{array}$ & $\begin{array}{l}\text { Yess } \\
\text { Yes }\end{array}$ & 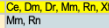 \\
\hline & 50p. (62633) & GT & Yes & $M m, R n$ & $104 \mathrm{p}(\mathrm{N} / 28 \mathrm{M})$ & $G \otimes A$ & Yes & $M n, B n, x t$ \\
\hline & 51p.,P2866L) & $C>T$ & Yes & $\mathrm{Mm}, \mathrm{R} \mathrm{n}$ & $10: p \cdot(R 74 \mathrm{~W})$ & $C D T$ & Yes & $M n, R n, \times t$ \\
\hline & 52p. (S273P) & $T x$ & Yes & $M m, R n$ & $10 \mathrm{pe}(\mathrm{p} / 55 \mathrm{l})$ & $\Theta \curvearrowright A$ & Yes & $\mathrm{Mn}, \mathrm{Rn}$ \\
\hline & 53p. (S276C) & $\begin{array}{l}\infty \rightarrow 6 \\
\text { CT }\end{array}$ & $\begin{array}{l}\text { Yes } \\
\text { Yes }\end{array}$ & $\begin{array}{ll}M m, R n \\
M n n\end{array}$ & 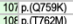 & $\begin{array}{l}C A \\
C D\end{array}$ & $\begin{array}{l}\text { Yes } \\
\text { Yyes }\end{array}$ & $\mathrm{Dr}, \mathrm{Mm}, \mathrm{Rn}, x$ \\
\hline & 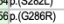 & $G A A$ & Yes & $M m, R n$ & 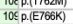 & $G>A$ & Yes & 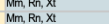 \\
\hline
\end{tabular}

\section{Acknowledgements}

This project was made possible through the help and guidance of MSc. Zeynep Oztürk of whom I am extremely appreciative to. I would also like to thank my parents for their endless support in my education.

\section{References}

1. Durmaz A, Kumral E, Durmaz B, Huseyin A., Aslan G., Ozkinay F., Pehlivan S., Orman M., Cogulu O. Genetic factors associated with the predisposition to late onset Alzheimer's disease. Gene. 2019;707:212-215. doi:10.1016/j.gene.2019.05.030 https:// pubmed ncbi.nlm.nih.gov/31102717/.

2.2020 Alzheimer's disease facts and figures [published online ahead of print,2020Mar 10].Alzheimers Dement.2020;10.1002alz.12068 doi:10.1002/alz.12068 https://alz-journals.onlinelibrary.wiley. com/doi/full/10.1002/alz.12068

3. Raji CA, Lopez OL, Kuller LH, Carmichael OT, Becker JT. Age, Alzheimer disease, and brain structure. Neurology. 2009;73(22):18991905. doi:10.1212/WNL.0b013e3181c3f293 https://pubmed.ncbi nlm.nih.gov/19846828/

4. "Alzheimer's Disease | AD.” MedlinePlus, U.S. National Library of Medicine, 8 June 2020, medlineplus.gov/alzheimersdisease.html https://medlineplus.gov/alzheimersdisease.html.

5. "Alzheimer Disease: MedlinePlus Medical Encyclopedia." Medlineplus.Gov, 2016, medlineplus.gov/ency/article/000760. htmlhttps://medlineplus.gov/ency/article/000760.htm

6. Bekris, Lynn M et al. "Genetics of Alzheimer disease." Journal of geriatric psychiatry and neurology vol. 23,4 (2010): 213-27. doi:10.1177/0891988710383571 https://www.ncbi.nlm.nih.gov pmc/articles/PMC3044597/

7. Barbier, Pascale et al. "Role of Tau as a Microtubule-Associated Protein: Structural and Functional Aspects." Frontiers in aging neuroscience vol. 11 204. 7 Aug. 2019, doi:10.3389/

fnagi.2019.00204 https://www.ncbi.nlm.nih.gov/pmc/articles/ PMC6692637/

8. Guo, T., Noble, W. \& Hanger, D.P. Roles of tau protein in health and disease. Acta Neuropathol 133, 665-704 (2017). https://doi.org/10.1007/s00401-017-1707-9. 
9. Iqbal K, Liu F, Gong CX, Grundke-Iqbal I. Tau in Alzheimer disease and related tauopathies. Curr Alzheimer Res. 2010; 7(8):656-664. doi:10.2174/156720510793611592

https://pubmed.ncbi.nlm.nih.gov/20678074/

10. "IARC TP53 Database." Amino Acid Properties, p53.iarc.fr AAProperties.aspx. https://p53.iarc.fr/AAProperties.aspx. Online Tools Geno2MP, https://geno2mp.gs.washington.edu/ Geno2MP/\#/MARRVEL, marrvel.org/human/gene/4137.

Microtubule-Associated Protein Tau Isoform 6

[Homo Sapiens] - Protein - NCBI. www.ncbi.nlm.nih.gov/ protein/NP_001116538.2.Embl-Ebi.“Clustal Omega.”EBI, www. ebi.ac.uk/Tools/msa/clustalo/.

\section{- Author}

Tejas Shah, the first and corresponding author of this paper, is a senior (Grade 12), currently attending Hopkinton High School. He is ever grateful for having been able to pursue a project that relates to his passion of entering the field of medicine. Tejas hopes to conduct similar research as an aspiring neuroscience major in the near future. 